\title{
Adaptive neural network synchronization for uncertain strick-feedback chaotic systems subject to dead-zone input
}

\section{Guanjun $\mathrm{Li}^{1 *}$ (B)}

\section{"Correspondence:}

lichampion2004@126.com

'Department of Applied

Mathematics, Huainan Normal

University, Huainan, China

\section{Springer}

\begin{abstract}
In this paper, an adaptive neural network (NN) synchronization controller is designed for two identical strict-feedback chaotic systems (SFCSs) subject to dead-zone input. The dead-zone models together with the system uncertainties are approximated by NNs. The dynamic surface control (DSC) approach is applied in the synchronization controller design, and the traditional problem of "explosion of complexity" that usually occurs in the backstepping design can be avoided. The proposed synchronization method guarantees the synchronization errors tend to an arbitrarily small region. Finally, this paper presents two simulation examples to confirm the effectiveness and the robustness of the proposed control method.
\end{abstract}

Keywords: Neural network; Chaos synchronization; Dead-zone

\section{Introduction}

Chaos synchronization (CS) has been widely investigated based on the results of [1]. A lot of works have been given on this theme because of its possible application in many fields such as communications, information processing [2-18]. For example, Liu et al. [19] discussed robust synchronization of uncertain complex networks by using impulsive control. $\mathrm{Yu}$ and $\mathrm{Cao}[20]$ addressed the synchronization of chaotic systems with time delay. The synchronization and anti-synchronization via active control approach on fractional chaotic financial system were studied by Huang et al. in [21]. On the basis of control theory, a lot of methods, such as adaptive control [22], sliding control [23,24], pinning control [25], intermittent control [26, 27], have been developed for CS.

In fact, it is known that system uncertainty is unavoidable in many practical applications, which always affects the control performance. On the other hand, fuzzy logic systems (FLSs) or neural networks (NNs) are usually employed to approximate unknown nonlinear functions. A major advantage of these systems is that they can be used to tackle nonlinear systems that have mismatching conditions and the uncertainties which are linearly parameterized. Therefore, approximation-based adaptive control based on NNs or FLSs is an interesting issue [28-36]. According to the NN model, backstepping control of an uncertain chaotic system is given in [37]. In [38], the author researches convergence for strict-feedback systems with functional uncertainties by using NN learning control methods.

(c) The Author(s) 2018. This article is distributed under the terms of the Creative Commons Attribution 4.0 International License (http://creativecommons.org/licenses/by/4.0/), which permits unrestricted use, distribution, and reproduction in any medium, provided you give appropriate credit to the original author(s) and the source, provide a link to the Creative Commons license, and indicate if changes were made. 
In addition, the dead zone and dynamic disturbances are the most important nonlinearities in most of industrial processes such as electric servomotors, hydraulic actuators, and motor. To tackle unknown dead zones and disturbances, some adaptive control techniques were proposed [39-42]. In [39], nonlinear systems with dead zone are studied based on dynamical surface control (DSC).

Generally speaking, there are two reasons to motivate the research of this paper. One is that, in the existing backstepping control of nonlinear systems, the problem "explosion of terms" was not well solved (for example, see [5]). The other is that the control of strict-feedback chaotic systems (SFCSs) subject to dead-zone input has been rarely investigated. This paper will propose the synchronization control methods for a class of uncertain SFCSs with dead-zone input and disturbances. The NN is used to approximate the unknown nonlinear function due to its good approximation performance. It should be emphasized that by using NN, the exact mathematical model of the controlled nonlinear systems can be unknown. By the Lyapunov function method, the synchronization error will remain in a small neighborhood of zero.

\section{Description of the NN}

A three-layer MIMO NN is employed to approximate an unknown continuous nonlinear function. The structure of this kind of NNs is depicted in Fig. 1.

Suppose that there are $\eta, k$, and $m$ neurons, then the mathematical model of the above $\mathrm{NN}$ is expressed by

$$
y_{p}\left(s, w_{p}\right)=\sum_{j=1}^{k} \omega_{p j} \varphi_{p j}\left(\sum_{i=1}^{\eta} v_{j i} s_{i}+\theta_{j}\right)=w_{p}^{T} \omega_{p}(\cdot),
$$

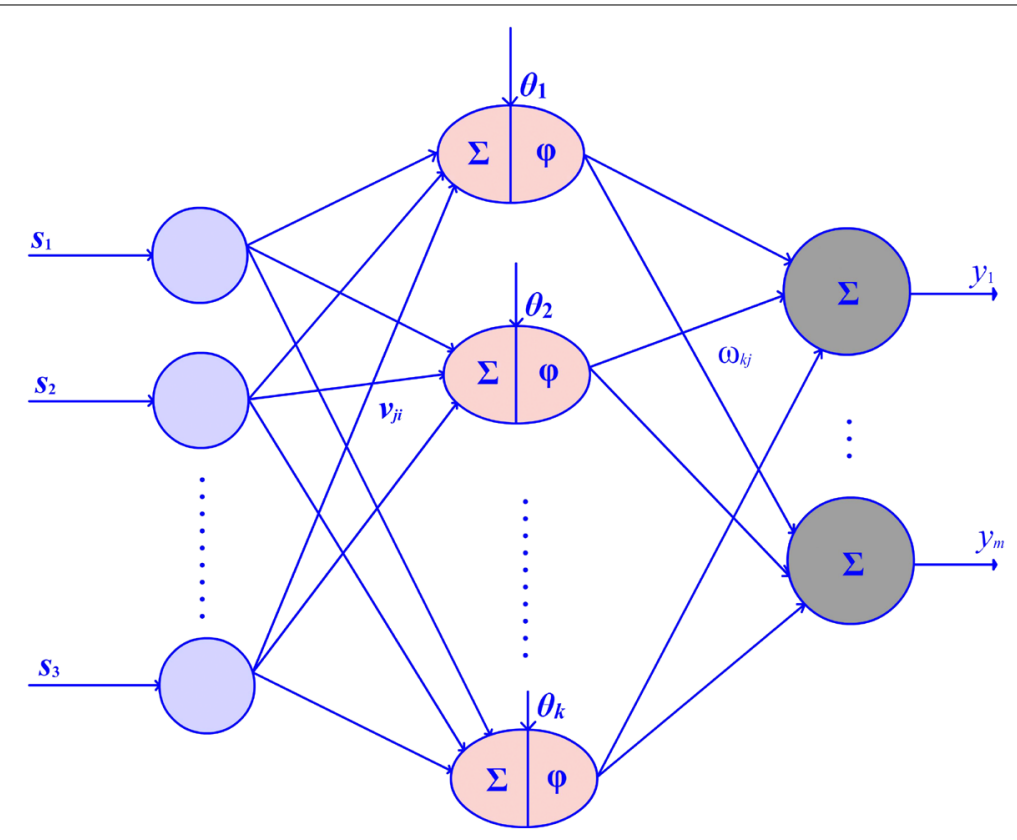

Figure 1 NN structure 
in which $p=1, \ldots, m$,

$$
w_{p}=\left[\begin{array}{c}
\omega_{p 1} \\
\vdots \\
\omega_{p k}
\end{array}\right], \quad \omega_{p}=\left[\begin{array}{c}
\varphi_{p 1}\left(\sum_{i=1}^{\eta} v_{1 i} s_{i}+\theta_{1}\right) \\
\vdots \\
\varphi_{p h}\left(\sum_{i=1}^{\eta} v_{h i} s_{i}+\theta_{k}\right)
\end{array}\right],
$$

$y_{k}$ represents the output. Usually, the function $\varphi(\cdot)$ is selected as

$$
\varphi(\xi)=\frac{e^{\xi}-e^{-\xi}}{e^{\xi}+e^{-\xi}} .
$$

Denote

$$
\varpi=\left[\begin{array}{c}
w_{1}^{T} \\
w_{2}^{T} \\
\vdots \\
w_{m}^{T}
\end{array}\right], \quad \vartheta=\left[\begin{array}{cccc}
\omega_{1}(\cdot) & 0 & \cdots & 0 \\
0 & \omega_{2}(\cdot) & \cdots & 0 \\
\vdots & \vdots & \ddots & \vdots \\
0 & 0 & \cdots & \omega_{m}(\cdot)
\end{array}\right] \quad \text { and } \quad y=\left[\begin{array}{c}
y_{1} \\
y_{2} \\
\vdots \\
y_{m}
\end{array}\right]
$$

NN (1) can be expressed by

$$
y=\varpi^{T} \vartheta .
$$

NNs (2) will be employed to estimate some unknown continuous nonlinear functions $f(x(t))$ in the next section as the form

$$
f(x(t))=\varpi^{* T} \vartheta(x(t))+\varepsilon(x(t)),
$$

where $\varepsilon(x(t))$ denotes the approximation error, and the ideal approximate parameter $\varpi^{*}$ is defined as

$$
\varpi^{*}=\arg \min _{\varpi}\left[\max \left|\varpi^{T} \vartheta(x(t))-f(x(t))\right|\right] .
$$

\section{Controller design and stability analysis}

\subsection{Problem description}

Consider the following uncertain SFCSs as the master system:

$$
\left\{\begin{array}{l}
\dot{y}_{k}(t)=f_{k}\left(\underline{y}_{k}(t)\right)+y_{k+1}(t), \quad k=1, \ldots, n-1, \\
\dot{y}_{n}(t)=f_{n}\left(\underline{y}_{n}(t)\right) \\
x(t)=y_{1}(t)
\end{array}\right.
$$

with $\underline{y}_{k}(t)=\left[y_{1}(t), \ldots, y_{k}(t)\right]^{T} \in \mathbb{R}^{k}, f_{k}(\cdot)$ is assumed to be unknown, and $x(t)$ is the output variable. The slave SFCS is given by

$$
\left\{\begin{array}{l}
\dot{\hat{y}}_{k}(t)=f_{k}(\hat{y}(t))+\hat{y}_{k+1}(t)+d_{k}(t), \quad k=1, \ldots, n-1, \\
\dot{\hat{y}}_{n}(t)=f_{n}\left(\hat{y}_{n}(t)\right)+u(t)+d_{n}(t), \\
\hat{x}(t)=\hat{y}_{1}(t),
\end{array}\right.
$$




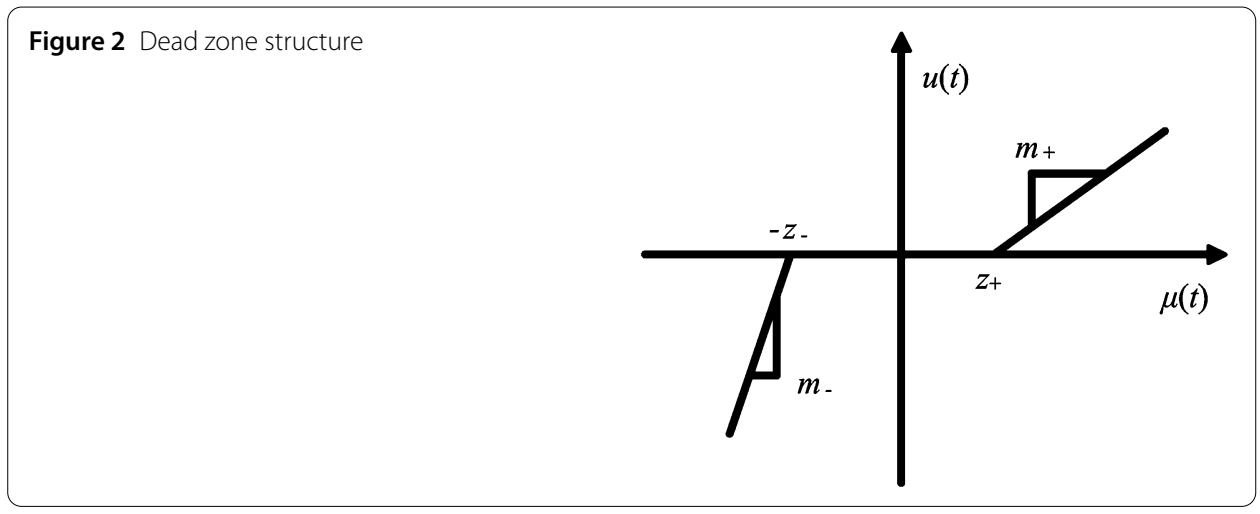

where $\underline{\hat{y}}_{k}(t)=\left[\hat{y}_{1}(t), \ldots, \hat{y}_{k}(t)\right]^{T} \in \mathbb{R}^{k}, u(t) \in \mathbb{R}$ is the control input and the output of the dead zone, $d_{k}(t)$ denotes an unknown external disturbance satisfying $\left|d_{k}(t)\right| \leq d_{k}^{*}$, where $d_{k}^{*}$ is a positive constant. The dead-zone input $u(t)$ is described as

$$
u(t)=D(\mu(t))= \begin{cases}m_{+}\left(\mu(t)-z_{+}\right), & \mu(t) \geq z_{+}, \\ 0, & -z_{-}<\mu(t)<z_{+}, \\ m_{-}\left(\mu(t)+z_{-}\right), & \mu(t) \leq-z_{-},\end{cases}
$$

where $\mu(t)$ denotes the dead-zone input, $m_{+}$and $m_{-}$respectively represent the right and left slopes, $z_{+}$and $z_{-}$are breakpoints. In addition, the above four parameters are all positive. This framework of the dead zone is depicted in Fig. 2.

The dead-zone input can be further modeled by

$$
u(t)=m(t) \mu(t)+b(t)
$$

where

$$
b(t)= \begin{cases}-m(t) z_{+}, & \text {if } \mu(t) \geq z_{+}, \\ -m(t) \mu(t), & \text { if }-z_{-}<\mu(t)<z_{+}, \\ m(t) z_{-}, & \text {if } \mu(t) \leq-z_{-},\end{cases}
$$

and

$$
m(t)= \begin{cases}m_{+}, & \text {if } \mu(t)>0 \\ m_{-}, & \text {if } \mu(t) \leq 0 .\end{cases}
$$

Remark 1 It should be mentioned that the dead-zone model (6) is representative because the conditions that $m_{+} \neq m_{-}$and $z_{+} \neq z_{-}$are involved. The dead-zone models were used in literature [43-45]. Besides, we know that $b(t)$ is a bounded function. In fact, denote $\bar{b}=\max \left\{m_{+} z_{+}, m_{+} z_{-}, m_{-} z_{+}, m_{-} z_{-}\right\}$, it follows from (8) that $|b(t)| \leq \bar{b}$.

\subsection{Synchronization controller implement}

In this part, we will give the detailed procedure of the adaptive $\mathrm{NN}$ controller design by using the backstepping control approach. The objective is to design aNN synchronization 
control such that the variable $\hat{x}(t)$ synchronizes the signal $x(t)$. The backstepping design procedure is given as follows.

Step 1. Define the synchronization error as

$$
e_{1}(t)=x(t)-\hat{x}(t)
$$

Introducing a virtual signal $\hbar_{1}(t)$, then the updated synchronization error is expressed by

$$
\zeta_{1}(t)=e_{1}(t)-\hbar_{1}(t)
$$

It follows from (4), (5), and (11) that

$$
\dot{\zeta}_{1}(t)=f_{1}\left(\underline{y}_{1}(t)\right)-f_{1}\left(\hat{y}_{1}(t)\right)+y_{2}(t)-\hat{y}_{2}(t)-d_{1}(t)-\dot{\hbar}_{1}(t) .
$$

Unlike the traditional backstepping control approach, in this paper, we will introduce a virtual signal $\xi_{2}(t)$ :

$$
\beta_{2} \dot{\xi}_{2}(t)+\xi_{2}(t)=\delta_{2}(t)
$$

in which $\xi_{2}(0)=\delta_{2}(0)$, where $\delta_{2}(t)$ is a signal to be designed and $\beta_{2}>0$ is a constant.

Define $e_{2}(t)=y_{2}(t)-\hat{y}_{2}(t)-\xi_{2}(t)$. Let

$$
\dot{\hbar}_{1}(t)=-\sigma_{1} \hbar_{1}(t)+\xi_{2}(t)-\delta_{2}(t)
$$

where $\sigma_{1}$ is a positive design parameter. As a result, we have

$$
\dot{\zeta}_{1}(t)=f_{1}\left(\underline{y}_{1}(t)\right)-f_{1}\left(\underline{\hat{y}}_{1}(t)\right)+y_{2}(t)-\hat{y}_{2}(t)-d_{1}(t)+\sigma_{1} \hbar_{1}(t)-\xi_{2}(t)+\delta_{2}(t) .
$$

Let

$$
\zeta_{2}(t)=e_{2}(t)-\hbar_{2}(t)
$$

Then we choose the signal $\delta_{2}(t)$ as

$$
\delta_{2}(t)=-\sigma_{1} e(t)-\hbar_{2}(t)-\frac{1}{2} \zeta_{1}(t) \hat{\Xi} \vartheta_{1}^{T}\left(e_{1}(t)\right) \vartheta_{1}\left(e_{1}(t)\right),
$$

where $\Xi=\left\|\varpi^{*}\right\|^{2}, \tilde{\Xi}=\Xi-\hat{\Xi}$ with $\hat{\Xi}$ being the estimation of $\Xi$.

Let

$$
g_{1}(t)=f_{1}\left(\underline{y}_{1}(t)\right)-f_{1}\left(\underline{\hat{y}}_{1}(t)\right) .
$$

By employing $\mathrm{NN}(2)$, the unknown function $g_{1}(t)$ can be approximated by

$$
g_{1}(t)=\varpi^{* T} \vartheta\left(e_{1}(t)\right)+\varepsilon\left(e_{1}(t)\right) .
$$


From (17), (18), and (19), we have

$$
\begin{aligned}
\dot{\zeta}_{1}(t)= & -\sigma_{1} \zeta_{1}(t)+\zeta_{2}(t)+\varpi^{* T} \vartheta_{1}\left(e_{1}(t)\right)+\varepsilon_{1}\left(e_{1}(t)\right) \\
& -d_{1}(t)-\frac{1}{2} \zeta_{1}(t) \hat{\Xi} \vartheta_{1}^{T}\left(e_{1}(t)\right) \vartheta_{1}\left(e_{1}(t)\right) .
\end{aligned}
$$

Step $k(k=2,3, \ldots, n-1)$. Let $e_{k}(t)=y_{k}(t)-\hat{y}_{k}(t)-\xi_{k}(t)$. Then the updated synchronization error is given as

$$
\zeta_{k}(t)=e_{k}(t)-\hbar_{k}(t)
$$

Differentiating $\zeta_{k}(t)$ yields

$$
\dot{\zeta}_{k}(t)=f_{k}\left(\underline{y}_{k}(t)\right)-f_{k}\left(\hat{y}_{k}(t)\right)+y_{k+1}(t)-\hat{y}_{k+1}(t)-d_{k}(t)-\dot{\xi}_{k}(t)-\dot{\hbar}_{k}(t) .
$$

The virtual signal $\xi_{k+1}(t)$ can be designed as

$$
\beta_{k+1} \dot{\xi}_{k+1}(t)+\xi_{k+1}(t)=\delta_{k+1}(t), \quad \xi_{k+1}(0)=\delta_{k+1}(0)
$$

Choosing

$$
\dot{\hbar}_{k}(t)=-\sigma_{k} \hbar_{k}(t)+\xi_{k+1}(t)-\delta_{k+1}(t)
$$

we have

$$
\begin{aligned}
\dot{\zeta}_{k}(t)= & f_{k}\left(\underline{y}_{k}(t)\right)-f_{k}\left(\hat{y}_{k}(t)\right)+y_{k+1}(t)-\hat{y}_{k+1}(t)-d_{k}(t) \\
& -\dot{\xi}_{k}(t)+\sigma_{k} \hbar_{k}(t)-\xi_{k+1}(t)+\delta_{k+1}(t) .
\end{aligned}
$$

Let $\zeta_{k+1}(t)=e_{k+1}(t)-\hbar_{k+1}(t)$, and the signal $\delta_{k+1}(t)$ is chosen as

$$
\delta_{k+1}(t)=-\sigma_{k} e_{k}(t)-\hbar_{k+1}(t)+\dot{\xi}_{k}(t)-\frac{1}{2} \zeta_{k}(t) \hat{\Xi} \vartheta_{k}^{T}\left(\underline{e}_{k}(t)\right) \vartheta_{k}\left(\underline{e}_{k}(t)\right)-\zeta_{k-1}(t),
$$

where $\underline{e}_{k}(t)=\left[e_{1}(t), \ldots, e_{k}(t)\right]^{T}$.

Substituting (26) into (25), we obtain

$$
\begin{aligned}
\dot{\zeta}_{k}(t)= & -\sigma_{k} \zeta_{k}(t)+\zeta_{k+1}(t)+\varpi^{* T} \vartheta_{k}\left(\underline{e}_{k}(t)\right)+\varepsilon_{k}\left(\underline{e}_{k}(t)\right) \\
& -d_{k}(t)-\frac{1}{2} \zeta_{k}(t) \hat{\Xi} \vartheta_{k}^{T}\left(\underline{e}_{k}(t)\right) \vartheta_{k}\left(\underline{e}_{k}(t)\right)-\zeta_{k-1}(t) .
\end{aligned}
$$

Step $n$. Let $e_{n}(t)=y_{n}(t)-\hat{y}_{n}(t)-\xi_{n}(t)$. Then the updated synchronization error is given as

$$
\zeta_{n}(t)=e_{n}(t)-\hbar_{n}(t)
$$

Differentiating $\zeta_{k}(t)$, we have

$$
\dot{\zeta}_{n}(t)=f_{n}\left(\underline{y}_{n}(t)\right)-f_{k}\left(\underline{\hat{y}}_{n}(t)\right)-u(t)-d_{n}(t)-\dot{\xi}_{n}(t)-\dot{\hbar}_{n}(t) .
$$


Choosing

$$
\dot{\hbar}_{n}(t)=-\sigma_{n} \hbar_{n}(t)
$$

we have

$$
\begin{aligned}
\dot{\zeta}_{n}(t)= & \varpi^{* T} \vartheta_{n}\left(\underline{e}_{n}(t)\right)+\varepsilon_{n}\left(\underline{e}_{n}(t)\right)-m(t) \mu(t)-b(t) \\
& -d_{n}(t)-\dot{\xi}_{n}(t)+\sigma_{n} \hbar_{n}(t) .
\end{aligned}
$$

The control law $\mu(t)$ is chosen as

$$
\mu(t)=\frac{1}{m(t)}\left[\sigma_{n} e_{n}(t)-\dot{\xi}_{n}(t)+\frac{1}{2} \zeta_{n}(t) \hat{\Xi} \vartheta_{n}^{T}\left(\underline{e}_{n}(t)\right) \vartheta_{n}\left(\underline{e}_{n}(t)\right)+\zeta_{n-1}(t)\right]
$$

Substituting (32) into (31), we have

$$
\begin{aligned}
\dot{\zeta}_{n}(t)= & -\sigma_{n} \zeta_{n}(t)+\varpi^{* T} \vartheta_{n}\left(\underline{e}_{n}(t)\right)+\varepsilon_{n}\left(\underline{e}_{n}(t)\right) \\
& -d_{n}(t)-\frac{1}{2} \zeta_{n}(t) \hat{\Xi} \vartheta_{n}^{T}\left(\underline{e}_{n}(t)\right) \vartheta_{n}\left(\underline{e}_{n}(t)\right)-\zeta_{n-1}(t)-b(t) .
\end{aligned}
$$

The adaptation law can be given as

$$
\dot{\hat{\Xi}}=\sum_{k=1}^{n}\left(\frac{\gamma}{2} \zeta_{k}^{2}\right) \vartheta_{k}^{T}\left(\underline{e}_{k}(t)\right) \vartheta_{k}\left(\underline{e}_{k}(t)\right)-\sigma_{0} \hat{\Xi}
$$

where $\gamma$ and $\sigma_{0}$ are design positive constants.

\subsection{Stability analysis}

Theorem 1 Consider SFCSs (4) and (5). If the virtual signal is given as (22) and (23), the control law is chosen as (32), the adaptive law is given as (34), then all signals are uniformly bounded, and the synchronization error remains in an arbitrarily small region of zero.

Proof Let

$$
V(t)=\frac{1}{2} \sum_{k=1}^{n} \zeta_{k}^{2}(t)+\frac{1}{2 \gamma} \tilde{\Xi}^{2}
$$

By differentiating $V$ along (20),(27), and (33), we can gain

$$
\begin{aligned}
\dot{V}(t)= & \sum_{k=1}^{n} \zeta_{k}(t) \dot{\zeta}_{k}(t)-\frac{1}{\gamma} \tilde{\Xi} \dot{\hat{\Xi}} \\
= & -\sum_{k=1}^{n} \sigma_{k} \zeta_{k}^{2}(t)+\sum_{k=1}^{n} \zeta_{k}(t) \varpi^{* T} \vartheta_{k}\left(\underline{e}_{k}(t)\right)+\sum_{k=1}^{n} \zeta_{k}(t) \varepsilon_{k}\left(\underline{e}_{k}(t)\right) \\
& -\sum_{k=1}^{n} \zeta_{k}(t) d_{k}(t)-\frac{1}{2} \sum_{k=1}^{n} \zeta_{k}^{2}(t) \hat{\Xi} \vartheta_{k}^{T}\left(\underline{e}_{k}(t)\right) \vartheta_{k}\left(\underline{e}_{k}(t)\right) \\
& -\zeta_{n}(t) b(t)-\frac{1}{\gamma} \tilde{\Xi} \dot{\hat{\Xi}} .
\end{aligned}
$$


By applying the following inequalities:

$$
\begin{aligned}
& \zeta_{k}(t) \varpi^{* T}(t) \vartheta_{k}\left(\underline{e}_{k}(t)\right) \leq \\
& \quad \leq \frac{1}{2} \zeta_{k}^{2}(t) \varpi^{* T} \varpi^{*} \vartheta_{k}^{T}\left(\underline{e}_{k}(t)\right) \vartheta_{k}\left(\underline{e}_{k}(t)\right)+\frac{1}{2} \\
& \quad \zeta_{k}^{2}(t) \Xi \vartheta_{k}^{T}\left(\underline{e}_{k}(t)\right) \vartheta_{k}\left(\underline{e}_{k}(t)\right)+\frac{1}{2}, \\
& \zeta_{k}(t) \varepsilon_{k}\left(\underline{e}_{k}(t)\right) \leq \frac{1}{2} \zeta_{k}^{2}(t)+\frac{1}{2} \varepsilon_{k}^{* 2}, \\
& \zeta_{k}(t) d_{k}(t) \leq \frac{1}{2} \zeta_{k}^{2}(t)+\frac{1}{2} d_{k}^{* 2} \\
& \zeta_{n}(t) b(t) \leq \frac{1}{2} \zeta_{n}^{2}(t)+\frac{1}{2} \bar{b}^{2}
\end{aligned}
$$

thus

$$
\begin{aligned}
\dot{V}(t)= & -\sum_{k=1}^{n}\left(\sigma_{k}-1\right) \zeta_{k}^{2}(t)+\frac{1}{2} \zeta_{n}^{2}(t)+\frac{1}{2} \sum_{k=1}^{n} \zeta_{k}^{2}(t) \tilde{\Xi} \vartheta_{k}^{T}\left(\underline{e}_{k}(t)\right) \vartheta_{k}\left(\underline{e}_{k}(t)\right) \\
& +\varrho_{1}-\frac{1}{\gamma} \tilde{\Xi} \hat{\Xi},
\end{aligned}
$$

where $\varrho_{1}=\frac{n}{2}+\frac{1}{2} \sum_{k=1}^{n} \varepsilon_{k}^{* 2}+\frac{1}{2} \sum_{k=1}^{n} d_{k}^{* 2}+\frac{1}{2} \bar{b}^{2}$.

Substituting (34) into (41), then

$$
\dot{V}(t) \leq-\sum_{k=1}^{n}\left(\sigma_{k}-1\right) \zeta_{k}^{2}(t)+\frac{1}{2} \zeta_{n}^{2}(t)+\varrho_{1}+\frac{\sigma_{0}}{\gamma} \tilde{\Xi} \hat{\Xi} .
$$

Note that

$$
\frac{\sigma_{0}}{\gamma} \tilde{\Xi} \hat{\Xi}=\frac{\sigma_{0}}{\gamma} \tilde{\Xi}(\Xi-\tilde{\Xi}) \leq-\frac{\sigma_{0}}{2 \gamma} \tilde{\Xi}^{2}+\frac{\sigma_{0}}{2 \gamma} \Xi^{2} .
$$

Then (42) can be rearranged as

$$
\begin{aligned}
\dot{V}(t) & \leq-\sum_{k=1}^{n}\left(\sigma_{k}-1\right) \zeta_{k}^{2}(t)+\frac{1}{2} \zeta_{n}^{2}(t)+\varrho_{1}-\frac{\sigma_{0}}{2 \gamma} \tilde{\Xi}^{2}+\frac{\sigma_{0}}{2 \gamma} \Xi^{2} \\
& \leq-\sum_{k=1}^{n}\left(\sigma_{k}-\frac{3}{2}\right) \zeta_{k}^{2}(t)+\varrho_{2}-\frac{\sigma_{0}}{2 \gamma} \tilde{\Xi}^{2} \\
& \leq-c V(t)+\varrho_{2}
\end{aligned}
$$

where $\varrho_{2}=\frac{\sigma_{0}}{2 \gamma} \Xi^{2}+\varrho_{1}, c=\min \left\{2 \sigma_{k}-3, \sigma_{0}\right\}$.

From (44), we have

$$
V(t) \leq\left(V\left(t_{0}\right)-\frac{\varrho_{2}}{c}\right) e^{-c\left(t-t_{0}\right)}+\frac{\varrho_{2}}{c} .
$$

Thus, according to (45), all signals are uniformly bounded and the synchronization error will remain in a small neighborhood of zero. 


\section{Simulation studies}

There are two examples included in this section.

\subsection{Example $\mathrm{A}$}

Let Duffing's system [14] be the master system:

$$
\left\{\begin{array}{l}
\dot{y}_{1}(t)=y_{2}(t) \\
\dot{y}_{2}(t)=y_{1}(t)-y_{1}^{3}(t)-0.15 y_{2}(t)+0.3 \cos (t) \\
x(t)=y_{1}(t)
\end{array}\right.
$$

with initial conditions $\left[y_{1}(0), y_{2}(0)\right]=[2,-1]$. The chaotic behavior of system $(46)$ can be seen in Fig. 3.

The slave system is given as:

$$
\left\{\begin{array}{l}
\dot{\hat{y}}_{1}(t)=\hat{y}_{2}(t)+d_{1}(t), \\
\dot{\hat{y}}_{2}(t)=\hat{y}_{1}(t)-\hat{y}_{1}^{3}(t)-0.15 \hat{y}_{2}(t)+0.3 \cos (t)+u(t)+d_{2}(t), \\
\hat{x}(t)=\hat{y}_{1}(t),
\end{array}\right.
$$

with initial conditions $\left[\widehat{y}_{1}(0), \widehat{y}_{2}(0)\right]=[-2,2]$. In the dead-zone model, let $m_{+}=m_{-}=2$, $z_{+}=z_{-}=2$. The structure of the dead zone is given in Fig. 4 .

There are two NNs used in the simulation. The input of the first $\mathrm{NN}$ is $e_{1}(t)$. We give five functions distributed on interval $[-5,5]$. The second one uses $e_{1}(t)$ and $e_{2}(t)$ as its input. For each input, the functions are defined the same as above. The parameters are $\beta_{2}=\beta_{3}=1.2, \sigma_{1}=\sigma_{2}=\sigma_{3}=1, \gamma=10, \sigma_{0}=0.1$. The initial conditions are given as $\xi_{2}(0)=$ $\delta_{2}(0)=\xi_{3}(0)=\delta_{3}(0)=0$.

Figures 5 and 6 show the results. Figure 5 gives the CS performance. It is shown that the output of the slave system tracks the output of the master system in a very short time.

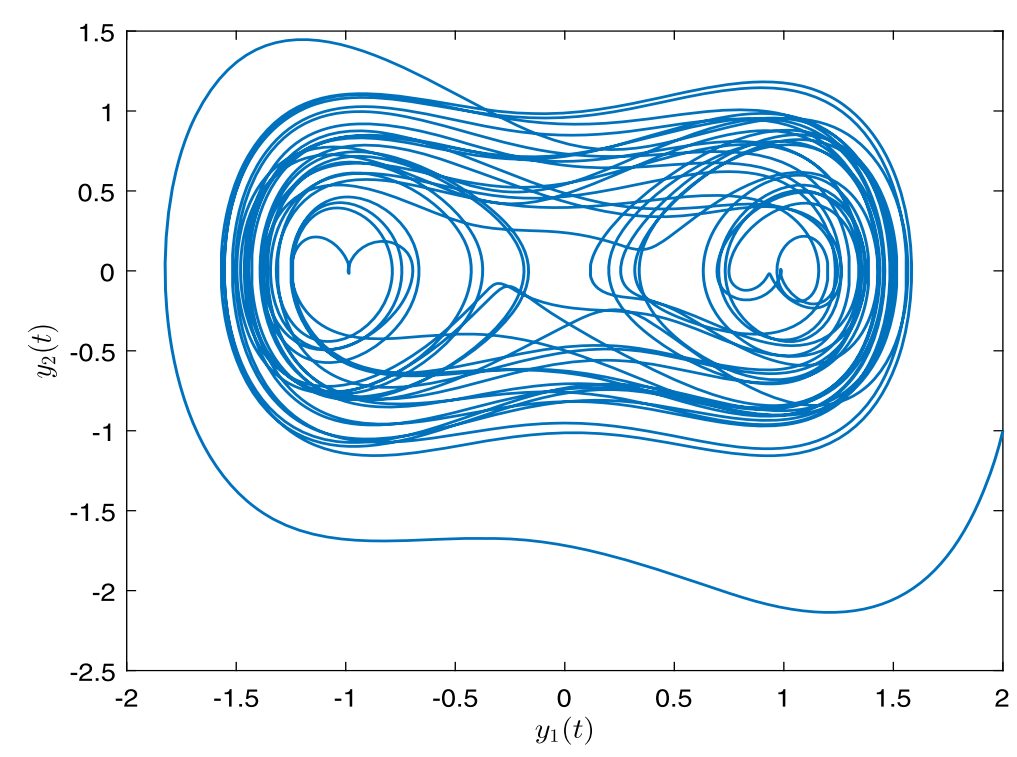

Figure 3 Chaotic behavior of system (46) 


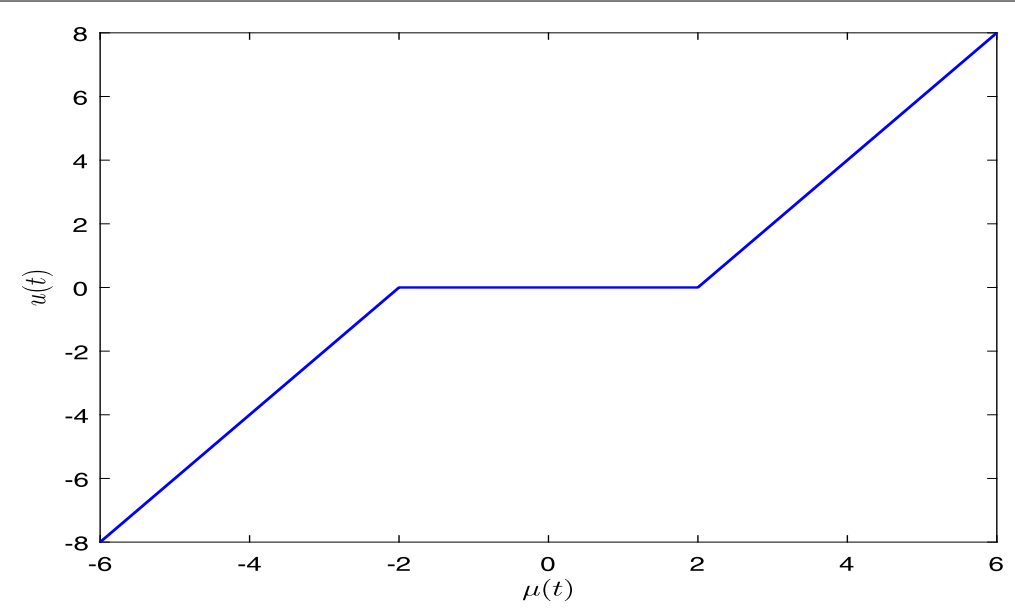

Figure 4 Structure of the dead-zone model

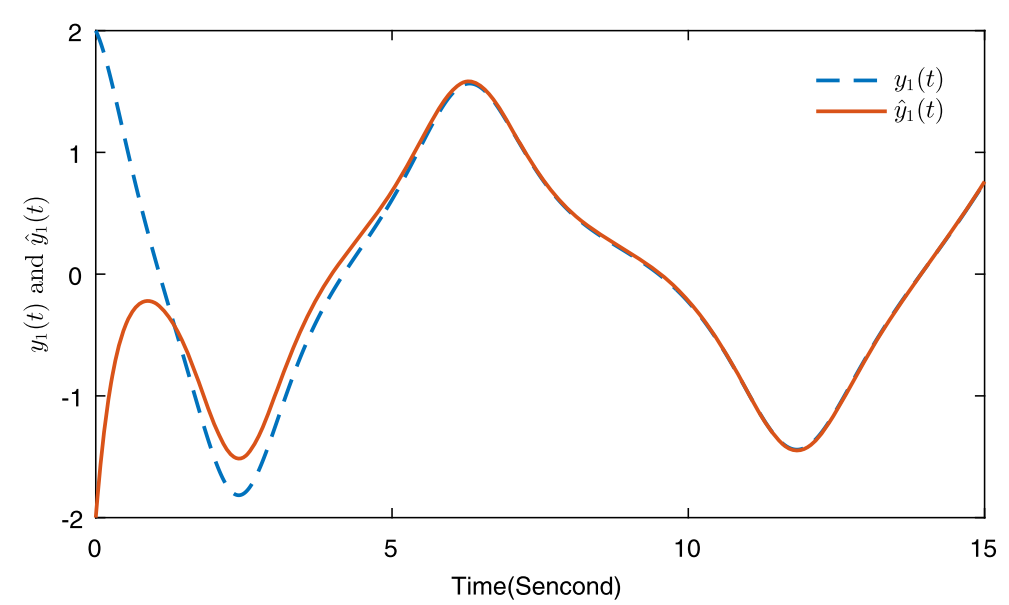

Figure 5 Synchronization performance

Time response of the control input, the dead zone, as well as the parameter of the NNs are given in Fig. 6.

\subsection{Example B}

Let Chua-Hartley's system be a master system:

$$
\left\{\begin{array}{l}
\dot{y}_{1}(t)=y_{2}(t)+\frac{10}{7}\left(y_{1}(t)-y_{1}^{3}(t)\right) \\
\dot{y}_{2}(t)=10 y_{1}(t)-y_{2}(t)+y_{3}(t), \\
\dot{y}_{3}(t)=-\frac{100}{7} y_{2}(t) \\
x(t)=y_{1}(t)
\end{array}\right.
$$

with initial conditions $\left[y_{1}(0), y_{2}(0), y_{3}(0)\right]=[-2,-1,1]$. The dynamical behavior of system (48) is given in Fig. 7. 

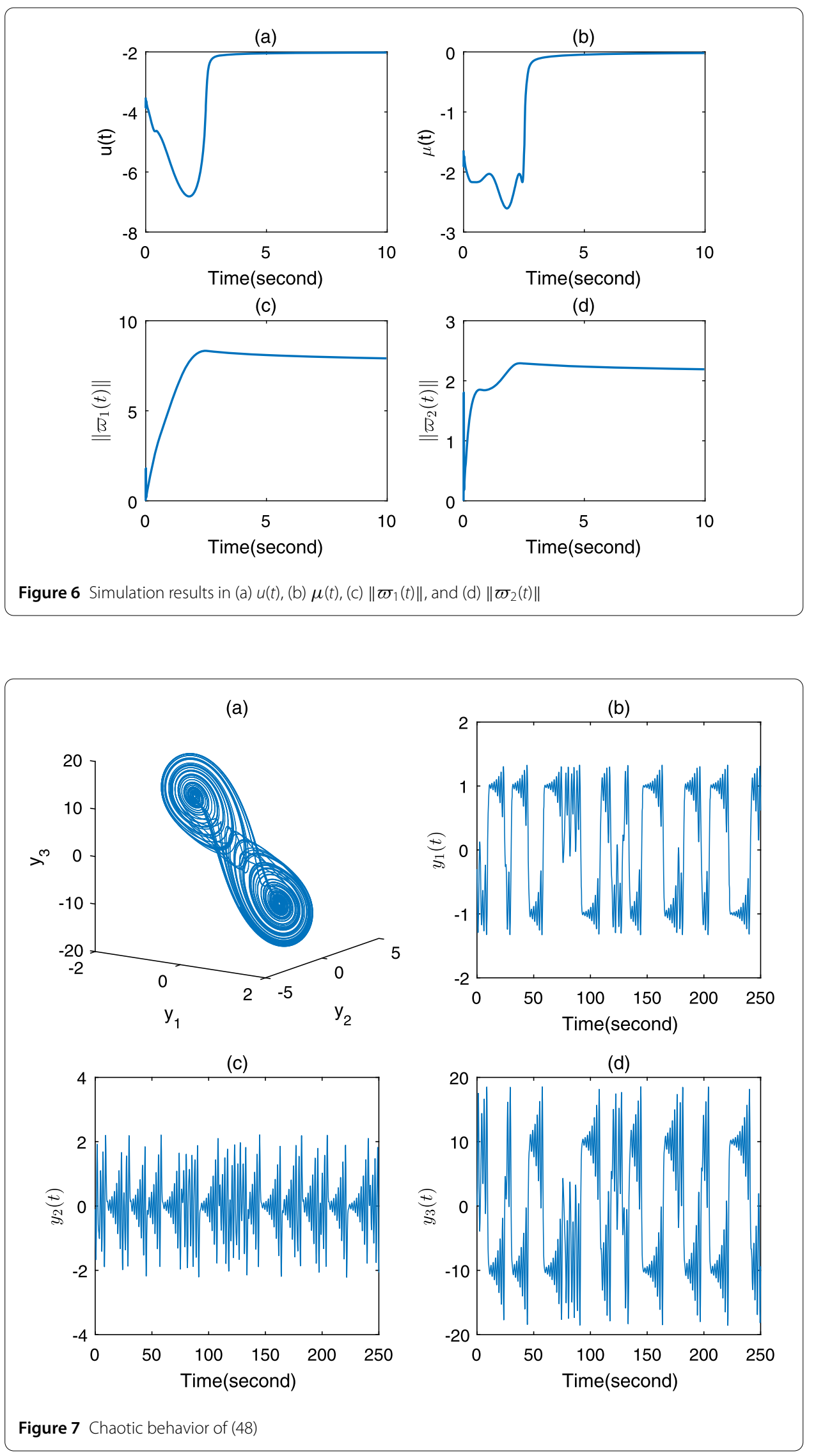

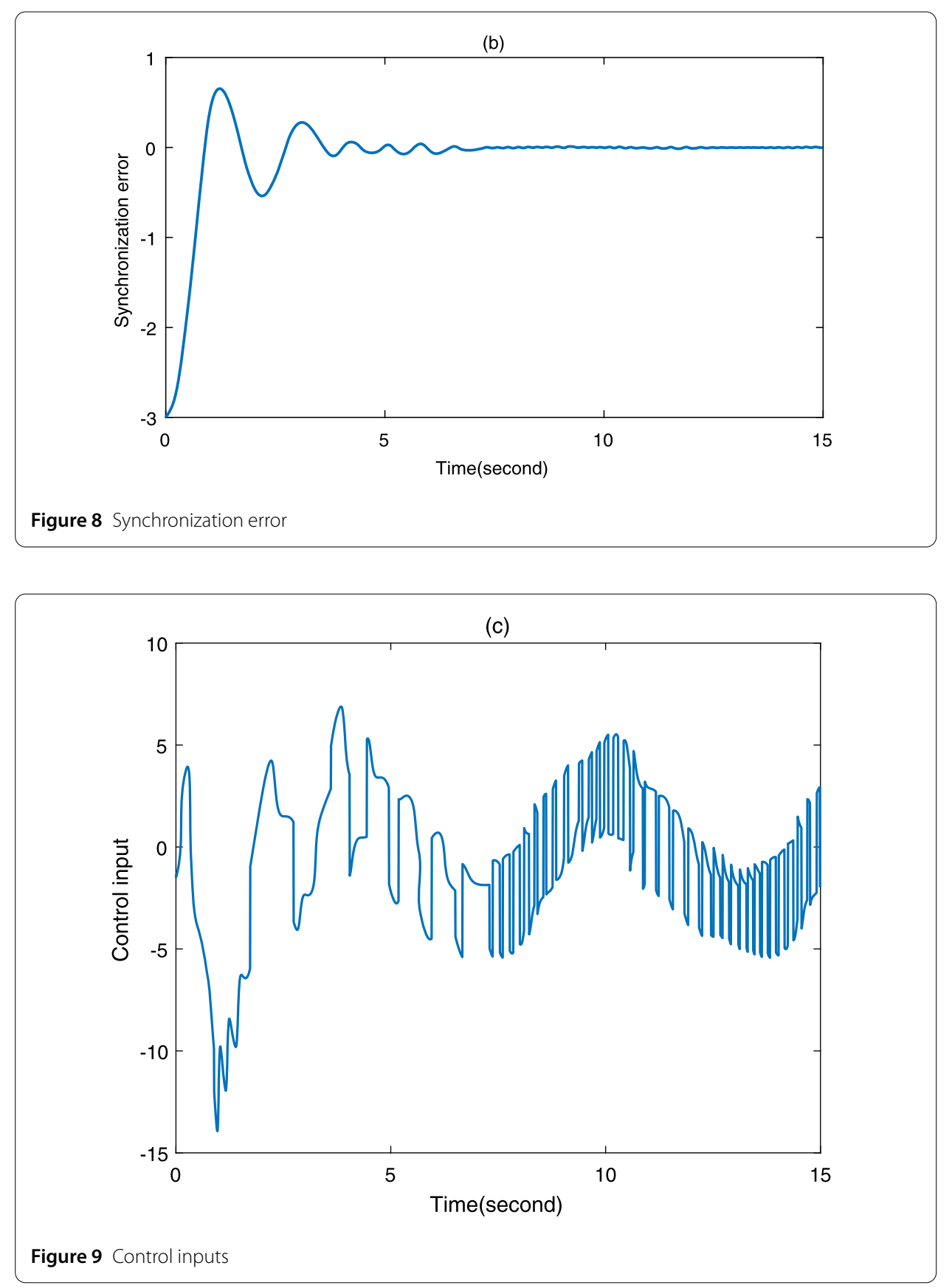

The slave system is given as:

$$
\left\{\begin{array}{l}
\dot{\hat{y}}_{1}(t)=\hat{y}_{2}(t)+\frac{10}{7}\left(\hat{y}_{1}(t)-\hat{y}_{1}^{3}(t)\right)+d_{1}(t) \\
\dot{\hat{y}}_{2}(t)=10 \hat{y}_{1}(t)-\hat{y}_{2}(t)+\hat{y}_{3}(t)+d_{2}(t) \\
\dot{y}_{3}(t)=-\frac{100}{7} \hat{y}_{2}(t)+u(t)+d_{3}(t) \\
\hat{x}(t)=\hat{y}_{1}(t)
\end{array}\right.
$$

with initial conditions $\left[\hat{y}_{1}(0), \hat{y}_{2}(0), \hat{y}_{3}(0)\right]=[2,2,-3]$. In the dead-zone model $m_{+}=m_{-}=2$, $z_{+}=z_{-}=2$. The control parameters are given as $\beta_{2}=\beta_{3}=\beta_{4}=1.2, \sigma_{1}=\sigma_{2}=\sigma_{3}=\sigma_{4}=1$, 


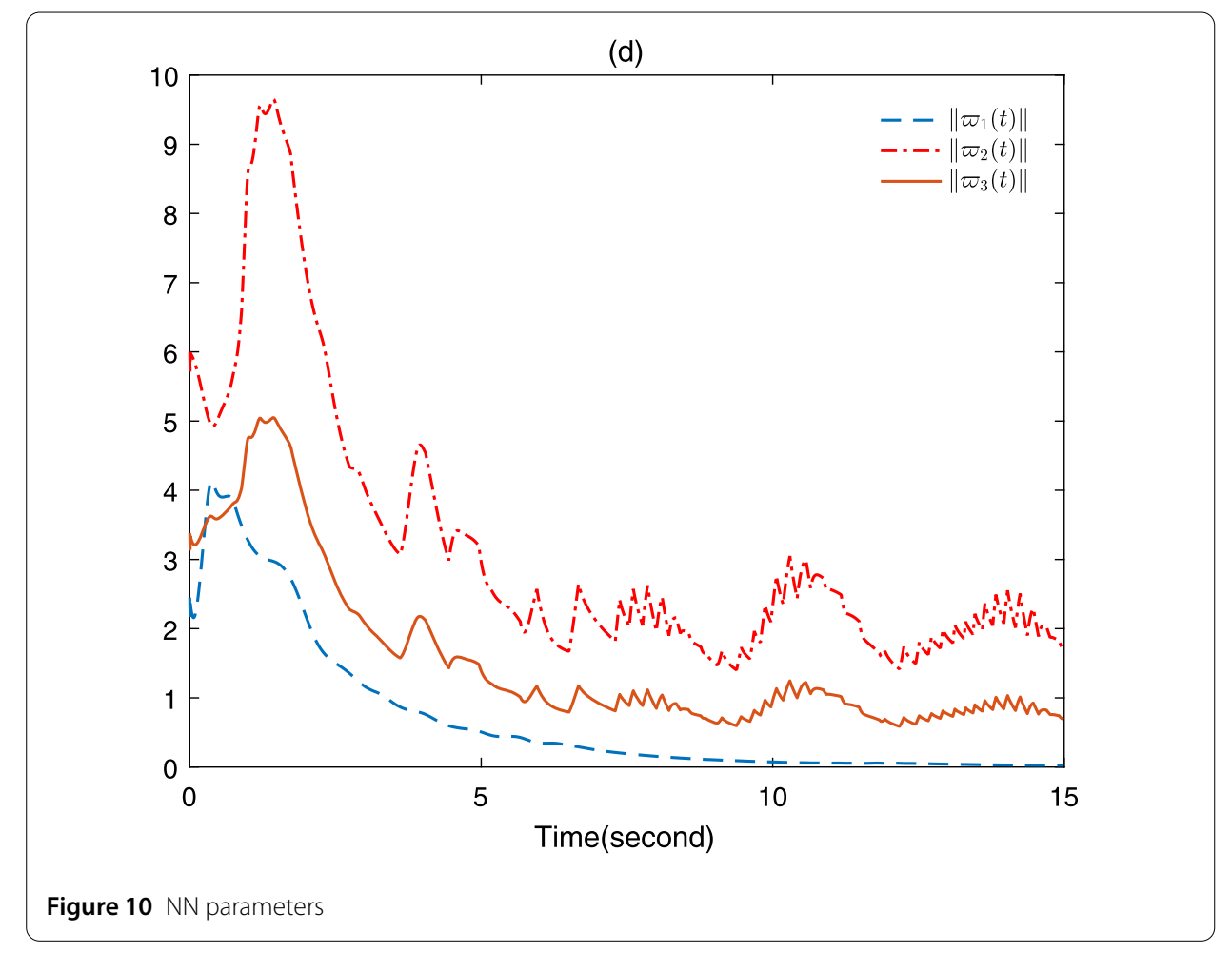

$\gamma=10, \sigma_{0}=0.2$. The initial conditions are given as $\xi_{2}(0)=\delta_{2}(0)=\xi_{3}(0)=\delta_{3}(0)=\xi_{4}(0)=$ $\delta_{4}(0)=0$.

There are three NNs included in the simulation. The first NN uses $e_{1}(t)$, the second NN uses $e_{1}(t)$ and $e_{2}(t)$, while the third NN uses $e_{1}(t), e_{2}(t)$, and $e_{3}(t)$ as their inputs, respectively. Let $\beta_{2}=\beta_{3}=\beta_{4}=1.2, \sigma_{1}=\sigma_{2}=\sigma_{3}=\sigma_{4}=1, \gamma=10, \sigma_{0}=0.1$. Our results are depicted in Figs. 8, 9, and 10, from which we can conclude that good synchronization performance has been achieved.

\section{Conclusions}

This paper provides a NN synchronization approach for SFCS with dead-zone input. It has been shown that the DSC approach has good ability to solve the "explosion of terms" problem in the backstepping control design.

Two simulation examples are given to confirm the proposed methods. How to extend the proposed control method to MIMO nonlinear systems is one of my future research directions.

Acknowledgements

This work is supported by the National Natural Science Foundation of China under Grant 11771263, and the Natural Science Foundation of Anhui Province of China under Grant 1808085MF181.

Competing interests

The author declares that there are no conflicts of interest regarding the publication of this paper.

Authors' contributions

All authors read and approved the final manuscript.

\section{Publisher's Note}

Springer Nature remains neutral with regard to jurisdictional claims in published maps and institutional affiliations. 
Received: 19 January 2018 Accepted: 8 May 2018 Published online: 18 May 2018

\section{References}

1. Pecora, L.M., Carroll, T.L.: Synchronization in chaotic systems. Phys. Rev. Lett. 64(8), 821-824 (1990)

2. Cao, J., Li, R.: Fixed-time synchronization of delayed memristor-based recurrent neural networks. Sci. China Inf. Sci. 60(3), $032201(2017)$

3. Wang, H., Liu, P.X., Liu, S.: Adaptive neural synchronization control for bilateral teleoperation systems with time delay and backlash-like hysteresis. IEEE Trans. Cybern. 47(10), 3018-3026 (2017)

4. Lu, J., Ho, D.W.C., Cao, J.: A unified synchronization criterion for impulsive dynamical networks. Automatica 46(7), $1215-1221(2010)$

5. Liu, H., Pan, Y., Li, S., Chen, Y.: Adaptive fuzzy backstepping control of fractional-order nonlinear systems. IEEE Trans. Syst. Man Cybern. Syst. 47, 2209-2217 (2017)

6. Bao, H.B., Cao, J.D.: Projective synchronization of fractional-order memristor-based neural networks. Neural Netw. 63, $1-9(2015)$

7. Li, G., Liu, H.: Stability analysis and synchronization for a class of fractional-order neural networks. Entropy 18(2), 55 (2016)

8. Das, S., Halder, U., Maity, D.: Chaotic dynamics in social foraging swarms-an analysis. IEEE Trans. Syst. Man Cybern., Part B, Cybern. 42(4), 1288-1293 (2012)

9. Sundarapandian, V., Sivaperumal, S.: Sliding controller design of hybrid synchronization of four-wing chaotic systems. Int. J. Soft Comput. 6(5-6), 224-231 (2011)

10. Liu, H., Li, S., Cao, J., Li, G., Alsaedi, A., Alsaadi, F.E.: Adaptive fuzzy prescribed performance controller design for a class of uncertain fractional-order nonlinear systems with external disturbances. Neurocomputing 219, 422-430 (2017)

11. Tu, Z., Cao, J., Alsaedi, A., Alsaadi, F.E., Hayat, T.: Global Lagrange stability of complex-valued neural networks of neutral type with time-varying delays. Complexity 21(S2), 438-450 (2016)

12. Liu, H., Li, S., Li, G., Wang, H.: Robust adaptive control for fractional-order financial chaotic systems with system uncertainties and external disturbances. Inf. Technol. Control 46, 246-259 (2017)

13. Liu, S., Zhou, L.: Network synchronization and application of chaotic Lur'e systems based on event-triggered mechanism. Nonlinear Dyn. 83(4), 2497-2507 (2016)

14. Liu, H., Chen, Y., Li, G., Xiang, W., Xu, G.: Adaptive fuzzy synchronization of fractional-order chaotic (hyperchaotic) systems with input saturation and unknown parameters. Complexity 2017, Article ID 6853826 (2017)

15. Cao, J., Sivasamy, R., Rakkiyappan, R.: Sampled-data $\mathrm{H}_{\infty}$ synchronization of chaotic Lur'e systems with time delay. Circuits Syst. Signal Process. 35(3), 811-835 (2016)

16. Chen, X., Cao, J., Ju, H.P., Huang, T., Qiu, J.: Finite-time multi-switching synchronization behavior for multiple chaotic systems with network transmission mode. J. Franklin Inst. 355(5), 2892-2911 (2018)

17. Boulkroune, A., Bouzeriba, A., Hamel, S., Bouden, T.: A projective synchronization scheme based on fuzzy adaptive control for unknown multivariable chaotic systems. Nonlinear Dyn. 78(1), 433-447 (2014)

18. Zhao, M., Liu, R., Gao, Y.: Dissipative lag synchronization of chaotic Lur'e systems with unknown disturbances. IMA J. Math. Control Inf. 34(1), 123-138 (2017)

19. Liu, B., Liu, X., Chen, G., Wang, H.: Robust impulsive synchronization of uncertain dynamical networks. IEEE Trans Circuits Syst. I, Regul. Pap. 52(7), 1431-1441 (2005)

20. Yu, W., Cao, J.: Adaptive synchronization and lag synchronization of uncertain dynamical system with time delay based on parameter identification. Physica A 375(2), 467-482 (2007)

21. Huang, C., Cao, J.: Active control strategy for synchronization and anti-synchronization of a fractional chaotic financial system. Physica A 473(2), 526-537 (2017)

22. Chen, X., Qiu, J., Cao, J., He, H.: Hybrid synchronization behavior in an array of coupled chaotic systems with ring connection. Neurocomputing 173, 1299-1309 (2016)

23. Liu, H., Pan, Y., Li, S., Chen, Y.: Synchronization for fractional-order neural networks with fullunder-actuation using fractional-order sliding mode control. Int. J. Mach. Learn. Cybern. (2017). https://doi.org/10.1007/s13042-017-0646-z

24. Chen, X., Ju, H.P., Cao, J., Qiu, J.: Sliding mode synchronization of multiple chaotic systems with uncertainties and disturbances. Appl. Math. Comput. 308, 161-173 (2017)

25. Yu, W., Chen, G., Lü, J., Kurths, J.: Synchronization via pinning control on general complex networks. SIAM J. Control Optim. 51(2), 1395-1416(2013)

26. Yang, X., Cao, J., Lu, J.: Stochastic synchronization of coupled neural networks with intermittent control. Phys. Lett. A 373(36), 3259-3272 (2009)

27. Cheng, L., Chen, X., Qiu, J., Lu, J., Cao, J.: Aperiodically intermittent control for synchronization of switched complex networks with unstable modes via matrix $\omega$-measure approach. Nonlinear Dyn. 2, 1-12 (2018)

28. Pan, Y., Liu, Y., Xu, B., Yu, H.: Hybrid feedback feedforward: an efficient design of adaptive neural network control. Neural Netw. 76, 122-134 (2015)

29. Wu, J., Chen, W., Li, J.: Fuzzy-approximation-based global adaptive control for uncertain strict-feedback systems with a priori known tracking accuracy. Fuzzy Sets Syst. 273(8), 1-25 (2015)

30. Tu, Z., Cao, J., Hayat, T.: Matrix measure based dissipativity analysis for inertial delayed uncertain neural networks. Neural Netw. 75, 47-55 (2016)

31. Pan, Y., Yu, H.: Biomimetic hybrid feedback feedforward neural-network learning control. IEEE Trans. Neural Netw. Learn. Syst. 28(6), 1481-1487 (2017)

32. Chen, W., Ge, S.S., Wu, J., Gong, M.: Globally stable adaptive backstepping neural network control for uncertain strict-feedback systems with tracking accuracy known a priori. IEEE Trans. Neural Netw. Learn. Syst. 26(9), 1842-1854 (2015)

33. Boulkroune, A., M'saad, M., Farza, M.: Adaptive fuzzy system-based variable-structure controller for multivariable nonaffine nonlinear uncertain systems subject to actuator nonlinearities. Neural Comput. Appl. 28(11), 3371-3384 (2017)

34. Li, G., Cao, J., Alsaedi, A., Ahmad, B.: Limit cycle oscillation in aeroelastic systems and its adaptive fractional-order fuzzy control. Int. J. Mach. Learn. Cybern. 2, 1-9 (2017) 
35. Liu, H., Li, S., Wang, H., Sun, Y.: Adaptive fuzzy control for a class of unknown fractional-order neural networks subject to input nonlinearities and dead-zones. Inf. Sci. 454-455, 30-45 (2018)

36. Liu, H., Li, S., Li, G., Wang, H.: Adaptive controller design for a class of uncertain fractional-order nonlinear systems: an adaptive fuzzy approach. Int. J. Fuzzy Syst. 20(2)، 366-379 (2018)

37. Lin, D., Wang, X., Nian, F., Zhang, Y.: Dynamic fuzzy neural networks modeling and adaptive backstepping tracking control of uncertain chaotic systems. Neurocomputing 73(16-18), 2873-2881 (2010)

38. Pan, Y., Sun, T., Liu, Y., Yu, H.: Composite learning from adaptive backstepping neural network control. Neural Netw. 95, 134-142 (2017)

39. Ponce, I.U., Bentsman, J., Orlov, Y., Aguilar, L.T.: Generic nonsmooth $\mathcal{H}_{\infty}$ output synthesis: application to a coal-fired boiler/turbine unit with actuator dead zone. IEEE Trans. Control Syst. Technol. 23(6), 2117-2128 (2015)

40. Zhang, T.P., Ge, S.S.: Adaptive dynamic surface control of nonlinear systems with unknown dead zone in pure feedback form. Automatica 44(7), 1895-1903 (2008)

41. Chen, X., Ju, H.P., Cao, J., Qiu, J.: Adaptive synchronization of multiple uncertain coupled chaotic systems via sliding mode control. Neurocomputing 273, 9-21 (2018)

42. Tong, S., Li, Y.: Adaptive fuzzy output feedback tracking backstepping control of strict-feedback nonlinear systems with unknown dead zones. IEEE Trans. Fuzzy Syst. 20(1), 168-180 (2012)

43. Yang, Z., Zhang, H.: A fuzzy adaptive tracking control for a class of uncertain strick-feedback nonlinear systems with dead-zone input. Neurocomputing 272, 130-135 (2017)

44. Wang, X.-S., Su, C.-Y., Hong, H.: Robust adaptive control of a class of nonlinear systems with unknown dead-zone. Automatica 40(3), 407-413 (2004)

45. Liu, Y.-J., Gao, Y., Tong, S., Li, Y.: Fuzzy approximation-based adaptive backstepping optimal control for a class of nonlinear discrete-time systems with dead-zone. IEEE Trans. Fuzzy Syst. 24(1), 16-28 (2016)

\section{Submit your manuscript to a SpringerOpen ${ }^{\circ}$ journal and benefit from:}

- Convenient online submission

- Rigorous peer review

- Open access: articles freely available online

- High visibility within the field

- Retaining the copyright to your article

Submit your next manuscript at $\gg$ springeropen.com 